\title{
Erratum: Steady-State Coherences by Composite System-Bath Interactions [Phys. Rev. Lett. 121, 070401 (2018)]
}

\author{
Giacomo Guarnieri®, Michal Kolár̆, and Radim Filip \\ (Received 9 July 2021; revised 13 August 2021; published 15 September 2021)
}

DOI: 10.1103/PhysRevLett.127.129901

The Sokhotski-Plemelj identity used in Eq. (34) of the Supplemental Material (hereby abbreviated with SM) of our Letter contains a sign mistake, and the correct formula is given by [see e.g., Eq. (121) of [1]]

$$
\int_{0}^{+\infty} d \tau e^{ \pm i \omega \tau}=\pi \delta(\omega) \pm i \mathcal{P} \frac{1}{\omega}
$$

Moreover, the long-time coefficient $\gamma_{1}(+\infty)$ [see non-numbered equation below Eq. (34) of the SM] is equal to zero only for a super-Ohmic spectral density, while in general one finds $\gamma_{1}(+\infty)=2 \pi J_{\text {eff }}(0, T)=\left.4 \pi \lambda T(\omega / \Omega)^{s-1}\right|_{\omega=0}$. This implies in particular that, for a bath described by a Ohmic spectral density (i.e., $s=1),\left.\gamma_{1}(+\infty)\right|_{s=1}=4 \pi \lambda T$. In the case of a subOhmic spectral density, one would rigorously need to introduce a positive low-frequency cutoff, in order for the bath autocorrelation function to be well defined. Taking this fact into account, one could verify that $\left.\gamma_{1}(+\infty)\right|_{s<1}$ would be the product of a large integral and of an appropriately small coupling strength $\lambda$. It can be shown that corrections to the SSC due to any finite value of $\left.\gamma_{1}(+\infty)\right|_{s}$ appear in higher order in the system-bath coupling. In light of the above corrections, and maintaining the same notation as in Eq. (3) of our Letter, the steady-state solutions for the SSC for the first model up to second order in the system-bath coupling [i.e., Eq. (2) of our Letter] becomes

$$
\bar{v}_{1}=-\frac{f_{1} f_{2}}{\omega_{0}}\left[\Delta_{1} \tanh \left(\frac{\omega_{0}}{2 T}\right)+4 \lambda \Omega \Gamma(s)+\Delta_{2}\right] .
$$

It is important to stress that our result, i.e., the expression for the steady-state coherence (SSC), does not depend on the Bloch-Redfield master equation approach. As explicitly shown in the second part of the manuscript and in Sec. E of the SM, in fact, SSC can be equivalently obtained by means of a different and independent approach, namely a perturbation expansion of the global Gibbs state in the system-bath coupling strength. In particular, it can be rigorously shown that the value for the SSC up to second order in the coupling coincides for the two above-mentioned perturbative approaches (see Sec. VIII of the Supplementary Information of Ref. [2]).

It is moreover worth emphasizing that, despite not representing the focus of this work, the corresponding value for the steady-state populations $\bar{v}_{3}$ can instead either be obtained through a second-order expansion of the global Gibbs state or through a fourth-order expansion of the Bloch-Redfield master equation. A second-order truncation of the latter, in fact, would only be able to provide the zeroth order solution for $\bar{v}_{3}$, thus leading to observed negativities of the Kossakowski matrix that precisely and solely reflect the absence of the second-order corrections induced by the SSC onto the populations (and therefore leading to potentially unphysical states outside the Bloch sphere). The reader is encouraged to check Refs. [2-4] for a thorough discussion in merit. We finally remark that the value for the second-order SSC Eq. (2) obtained through a fourth-order expansion of the Bloch-Redfield master equation would remain unchanged.

The correct result Eq. (2) indicates that, up to second order, the SSC is linear in both $f_{1}$ and $f_{2}$. Consequently, the spikes observed in Fig. 2(a) of our Letter disappear and no optimization over $f_{2}$ can be meaningfully performed. Equation (4) of our Letter is therefore to be neglected. The corrected version of the plot is provided in Fig. 1(a), which shows the quantity $\mathcal{C} /\left(f_{1} f_{2}\right)$ as a function of the bath temperature.

Similarly, the change of sign in Eq. (1) leads to the following result for the SSC in the second model considered, i.e., the one described by the Hamiltonian Eq. (5) of our Letter:

$$
\mathcal{C}=\left|\frac{2 f_{1} f_{2}}{\omega_{0}}\left[\delta_{1} \tanh \left(\frac{\omega_{0}}{2 T}\right)+\lambda \Omega \Gamma(s)-\delta_{2}\right]\right|,
$$



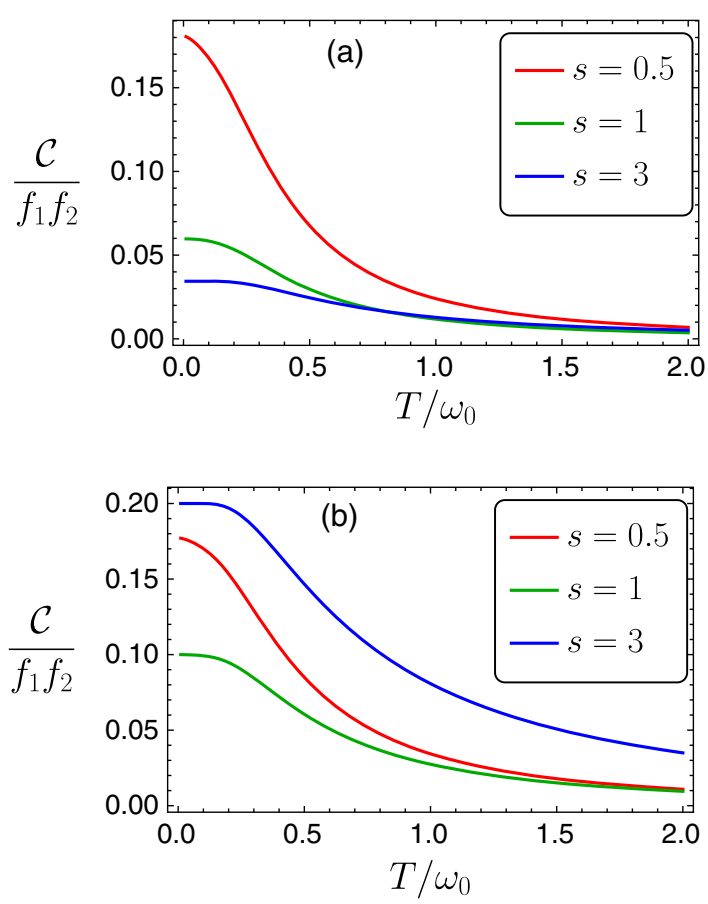

FIG. 1. Temperature profile of the $l_{1}$ norm of coherence in units of the system-bath couplings $f_{1,2}$. All the other parameters have been chosen as in our Letter. In particular, (a) refers to the first model, i.e., the one described by the Hamiltonian in Eq. (1) of our Letter, while (b) refers to the second model, i.e., Eq. (5) of our Letter.

where we have once again left the notation and definition of the various quantities unchanged and where we have furthermore discarded all the contributions of higher order than second. This expression amends Eq. (78) of the SM of our Letter and its behavior is shown in Fig. 1(b) [which thus substitutes Fig. 2(b) of our Letter].

Finally, expression (102) of the SM contains a typo in the last two terms: instead of being $-\sinh \left(\beta \omega_{0} y\right)+\sinh \left(\beta \omega_{0} x\right)$ it should be $-\sinh \left(\beta \omega_{0} x\right)+\sinh \left(\beta \omega_{0} y\right)$.

While some of us were working on the Letter, we became aware of all the above technical issues and, in fact, all the above correct expressions could also be found in Ref. [2]. In parallel we were also recently contacted by other colleagues who found the same gaps, and contributed to the present Erratum. We would therefore like to thank A. Purkayastha, G. Manzano, M. Cattaneo, A. Slobodeniuk, and T. Novotný, for insightful discussions and comments on the above results.

[1] M. Esposito, U. Harbola, and S. Mukamel, Rev. Mod. Phys. 81, 1665 (2009).

[2] A. Purkayastha, G. Guarnieri, M. T. Mitchison, R. Filip, and J. Goold, npj Quantum Inf. 6, 27 (2020).

[3] C. H. Fleming and N. I. Cummings, Phys. Rev. E 83, 031117 (2011).

[4] D. Tupkary, A. Dhar, M. Kulkarni, and A. Purkayastha, arXiv:2105.12091. 